\title{
A farnesoic acid O-methyltransferase (FAMeT) from Exopalaemon carinicauda is responsive to Vibrio anguillarum and WSSV challenge
}

\author{
Yafei Duan • Ping Liu • Jitao Li • Yun Wang • Jian Li • \\ Ping Chen
}

Received: 15 August 2013 /Revised: 12 September 2013 / Accepted: 13 September 2013 /Published online: 19 October 2013

(C) The Author(s) 2013. This article is published with open access at Springerlink.com

\begin{abstract}
Methyl farnesoate (MF), an analogue of the insect juvenile hormone III, is believed to play important roles in the regulation of the growth and reproductive development in crustaceans. Farnesoic acid O-methyltransferase (FAMeT) is the key enzyme in the juvenile hormone biosynthetic pathway, involved in the conversion of farnesoic acid (FA) to MF in the final step of MF synthesis. In this study, a FAMeT cDNA (named EcFAMeT) was cloned from the hemocytes of ridgetail white prawn Exopalaemon carinicauda by rapid amplification of cDNA ends (RACE) methods. The fulllength cDNA of EcFAMeT was $1,620 \mathrm{bp}$, including contains a $5^{\prime}$-untranslated region (UTR) of $75 \mathrm{bp}, 3^{\prime}$-UTR of $714 \mathrm{bp}$ with a poly (A) tail, an open reading frame (ORF) of $831 \mathrm{bp}$, encoding a 276-amino-acid polypeptide with the predicted molecular weight of $31.57 \mathrm{kDa}$ and estimated isoelectric point of 4.67. BLAST analysis revealed that amino acids of EcFAMeT shared high identity (75-90\%) with that of other crustaceans. Two conserved signatures domains of Methyltransf-FA superfamily were also identified in EcFAMeT. Real time quantitative RT-PCR analysis indicated that EcFAMeT could be detected in all the tested tissues and strongly expressed in hepatopancreas and ovary of $E$. carinicauda. After Vibrio anguillarum and WSSV challenge, EcFAMeT transcripts both in hemocytes and hepatopancreas increased significantly in the first $3 \mathrm{~h}$, respectively. The results
\end{abstract}

Y. Duan $\cdot$ P. Liu $(\bowtie) \cdot J . \mathrm{Li} \cdot$ J. Li $\cdot$ P. Chen

Key Laboratory of Sustainable Development of Marine Fisheries, Ministry of Agriculture, Yellow Sea Fisheries Research Institute, Chinese Academy of Fishery Sciences, Qingdao 266071, People's Republic of China

e-mail: liuping@ysfri.ac.cn

Y. Duan $\cdot$ Y. Wang

Key Laboratory of South China Sea Fishery Resources Exploitation \& Utilization, Ministry of Agriculture, South China Sea Fisheries Research Institute, Chinese Academy of Fishery Sciences, Guangzhou 510300, People's Republic of China indicated that EcFAMeT might be associated with the immune defenses to V. anguillarum and WSSV in E. carinicauda.

Keywords Exopalaemon carinicauda $\cdot$ Farnesoic acid O-methyltransferase (FAMeT) · Gene cloning · Vibrio anguillarum $\cdot$ WSSV

\section{Introduction}

The ridgetail white prawn Exopalaemon carinicauda is an economically important shrimp species naturally distributed in the coasts of the Yellow Sea and the Bohai Sea, China, which contributes to one third of the gross output of the polyculture ponds in eastern China (Xu et al. 2010). E. carinicauda have good reproductive performance, fast growth, and wide environmental adaptability, which make it a good experimental animal candidate for shrimp (Xu et al. 2010; Li et al. 2012; Wang et al. 2013). Due to its commercial value, "milky shrimp" disease caused by Hematodinium (Xu et al. 2010), immune gene discovery by expressed sequence tags (ESTs; Duan et al. 2013b) and identification of immunerelated genes such as heat shock protein (HSP 90; Li et al. 2012), selenium dependent glutathione peroxidase (GPx; Duan et al. 2013a), and peritrophin (Wang et al. 2013) have been studied in E. carinicauda. However, with the development of intensive culture and the ecologic environmental deterioration, various diseases caused by bacteria and viruses have frequently occurred in cultured E. carinicauda, causing economic losses to commercial shrimp aquaculture (Xu et al. 2010). Shrimp lack an adaptive immune system and their defense mechanisms mainly rely on innate immune responses to protect them against invaders. Therefore, a better understanding of the innate immune abilities and immune defense mechanisms of shrimp will be beneficial to the development 
of health management and disease control in shrimp aquaculture.

O-methyltransferase (OMT) is an enzyme that is ubiquitously present in diverse organisms and catalyses the methylation of both small and macromolecules for various functional and regulatory purposes (Li et al. 2006). OMT may play an important regulatory role in plant and animal growth, development, reproduction and defence, and is also implicated in human disease ( $\mathrm{Li}$ et al. 2006; Chiron et al. 2000; Yao et al. 2003; Fan et al. 2005). In invertebrates, most studies of the kinds of OMT focus on farnesoic acid O-methyltransferase (FAMeT). FAMeT is the enzyme responsible for the conversion of farnesoic acid (FA) to methyl farnesoate (MF) in the final step of MF synthesis using the cofactor S-adenosyl-L-methionine (SAM; Burtenshaw et al. 2008; Silva Gunawardene et al. 2003; Hui et al. 2008). MF is a sesquiterpenoid, structurally similar to insect juvenile hormone III (JH III) and is an unepoxidized analogue of JH III, deemed the juvenile hormone of crustaceans (Silva Gunawardene et al. 2001, 2003; Hui et al. 2008; Holford et al. 2004). Previous studies have demonstrated that MF play a vital role in the regulation of growth and reproductive development, including morphogenesis, metamorphosis, reproduction, and molting, and the response to stress in crustaceans (Silva Gunawardene et al. 2001; Holford et al. 2004; Averbeck et al. 2000). The catalytic activity of FAMeT is thought to occur at a rate-limiting step in the JH biosynthetic pathway although its exact role is yet to be defined (Williamson et al. 2001).

The first study of FAMeT was in Metapenaeus ensis (Silva Gunawardene et al. 2001). To date, the FAMeT gene has also been isolated from some crustaceans (Silva Gunawardene et al. 2001, 2003; Hui et al. 2008; Holford et al. 2004; Yang et al. 2012; Kuballa et al. 2007; Ruddell et al. 2003). Research on FAMeT has mainly focused on the importance of this enzyme and its regulation of MF production (Hui et al. 2008). However, no studies on FAMeT in E. carinicauda and the gene in response to Vibrio anguillarum and white spot syndrome virus (WSSV) have been reported. $V$. anguillarum and WSSV caused the most serious disease leading to major losses in the shrimp aquaculture industry around the world (Toranzo et al. 2005; Lightner 2011; Spann and Lester 1997), but there are still no effective preventive and therapeutic measures against $V$. anguillarum and WSSV infection.

The present study isolated and characterized the full-length cDNA from hemocytes of E. carinicauda, compared its sequence with other known FAMeTs, investigated the expression pattern of FAMeT in various tissues of E. carinicauda, and evaluated this FAMeT expression in hemocytes and hepatopancreas of $E$. carinicauda after $V$. anguillarum and WSSV challenge. These results will be essential to better understand the physiological function of FAMeT in the shrimp immune response to bacterial and viral infection.

\section{Materials and methods}

Animal materials

Healthy adult $E$. carinicauda, averaging weight $1.19 \pm 0.32 \mathrm{~g}$, were collected from a commercial farm in Qingdao, China. They were cultured in filtered aerated seawater (salinity $20 \%$, $\mathrm{pH} 8.2$ ) at $18 \pm 0.5^{\circ} \mathrm{C}$ for 7 days before processing. There were 30 prawns in each group. The prawns were fed daily with a ration of $10 \%$ of body weight, and two-thirds of the water in each group was renewed once daily.

\section{RNA extraction and cDNA synthesis}

Hemocytes were collected with a syringe which contained an equal volume of anticoagulant solution (Söderhäll and Smith 1983), and centrifuged at $800 \times g, 4{ }^{\circ} \mathrm{C}$ for $15 \mathrm{~min}$. Total RNA was extracted from hemocytes using Trizol Reagent (Invitrogen, USA) following the manufacturer's protocol. The RNA samples were analyzed in $1.0 \%$ agarose electrophoresis and quantitated at $260 \mathrm{~nm}$, all $\mathrm{OD}_{260} / \mathrm{OD}_{280}$ were between 1.8 and 2.0. The $3^{\prime}$ and $5^{\prime}$ ends RACE cDNA templates were synthesized using SMART ${ }^{\mathrm{TM}}$ cDNA Kit (Clontech, USA) following the protocol of the manufacturer.

\section{Cloning the full-length cDNA of EcFAMeT}

An EST sequence was obtained from E. carinicauda hemocytes cDNA library of our laboratory (GenBank accession no. JK996877) and has been reported by Duan et al. (2013b). BLAST analysis showed that they had high identifies with FAMeTs of other shrimps. According to the EST sequence, a gene specific primer F1 was designed for 3' RACE, and R1 was designed for $5^{\prime}$ RACE (Table 1).

Based on the EST sequence data of FAMeT, its $3^{\prime}$ and $5^{\prime}$ ends were obtained using SMART RACE cDNA Amplification Kit (Clontech, USA). For 3' RACE, the PCR reaction was performed using the primer $\mathrm{F} 1$ and the anchor primer UPM (Table 1). The PCR reaction conditions were 5 cycles of $94^{\circ} \mathrm{C}$ for $30 \mathrm{~s}, 72^{\circ} \mathrm{C}$ for $3 \mathrm{~min}, 5$ cycles of $94{ }^{\circ} \mathrm{C}$ for $30 \mathrm{~s}, 70^{\circ} \mathrm{C}$ for $30 \mathrm{~s}$, and $72{ }^{\circ} \mathrm{C}$ for $3 \mathrm{~min}$, and 25 cycles of $94{ }^{\circ} \mathrm{C}$ for $30 \mathrm{~s}, 68^{\circ} \mathrm{C}$ for $30 \mathrm{~s}$, and $72{ }^{\circ} \mathrm{C}$ for $3 \mathrm{~min}$. For $5^{\prime}$ RACE, the PCR reaction was performed using the primer R1 and the anchor primer UPM (Table 1). The PCR reaction conditions were the same as those described above.

The PCR fragments were subjected to electrophoresis on $1.0 \%$ agarose gel to determine length differences, and the target band was purified by PCR purification kit (Promega, USA). The purified products were cloned into PMD18-T vector, following the instructions provided by the manufacturer (TaKaRa, Japan). Recombinant bacteria were identified by blue/white screening and confirmed by PCR. Plasmids 
Table 1 Primer sequences used in this study

\begin{tabular}{ll}
\hline Primer name & Sequence $\left(5^{\prime}-3^{\prime}\right)$ \\
\hline EcFAMeT & \\
F1 (forward) & TGAAATCAGAGTCGGGAA \\
R1 (reverse) & GTTTTCCCAACCACCAAT \\
F2 (forward) & CTCAGGTTCCAGGTCAAAGC \\
R2 (reverse) & TCCTCATCACAGCACAGAGC \\
UPM & CTAATACGACTCACTATAGGGCAA \\
& GCAGTGGTATCAACGCAGAGT \\
& CTAATACGACTCACTATAGGGC \\
18S rRNA & \\
18S-HF & TATACGCTAGTGGAGCTGGAA \\
\hline
\end{tabular}

containing the insert were purified (Promega minipreps) and used as a template for DNA sequencing.

Sequence analysis

The nucleotide and deduced amino acid sequences of EcFAMeT cDNA were analyzed and compared using the BLAST search programs (http://www.blast.ncbi.nlm.nih.gov/ Blast.cgi). The signal peptide was predicted by SignalP program (http://www.cbs.dtu.dk/services/SignalP/). The multiple sequence alignment of FAMeT amino acid sequences was performed using the programs of Vector NTI advance 10.3 (Invitrogen). A phylogenetic NJ tree of FAMeTs was constructed by the MEGA 4.0 software (Tamura et al. 2007).

\section{Tissue expression of EcFAMeT}

Hemocytes, gill, hepatopancreas, muscle, ovary, eyestalk, stomach, and intestine were dissected from unchallenged $E$. carinicauda. The mRNA expressions of EcFAMeT in different tissues were determined by quantitative real-time RT-PCR. Total RNA was extracted as described above. The RNA samples were analyzed in $1.0 \%$ agarose electrophoresis and quantitated at $260 \mathrm{~nm}$, all $\mathrm{OD}_{260} / \mathrm{OD}_{280}$ were between 1.8 and 2.0. Total RNA $(5 \mu \mathrm{g})$ was reverse transcribed using the PrimeScript $^{\mathrm{TM}}$ Real time PCR Kit (TaKaRa, Japan) for realtime quantitative RT-PCR analysis. 18S rRNA gene of $E$. carinicauda (GenBank accession number: GQ369794) was used as an internal control.

Experimental design of $V$. anguillarum and WSSV challenge

The experiments were divided into the bacterial ( $V$. anguillarum) challenged group, the virus (WSSV) challenged group and the control group. $V$. anguillarum strains was obtained from Germplasm Resources and Genetic Breeding Laboratory, Yellow Sea Fisheries Research Institute (China), activating on marine agar 2611E. WSSV crude extract was obtained from $10 \mathrm{~g}$ WSSV-infected tissue from Litopenaeus vannamei, which was provided by the Mariculture Disease Control and Pathogenic Molecular Biology Laboratory, Yellow Sea Fisheries Research Institute, according to the methods of Xie et al. (2005). In the experiment, the challenged groups were injected individually with $20 \mu \mathrm{L}$ live $V$. anguillarum suspended in sterile $0.9 \%$ normal saline $(2 \times$ $10^{8} \mathrm{CFU} / \mathrm{mL}$ ) or $20 \mu \mathrm{L}$ live WSSV crude extract, and the control group received individually an injection of $20 \mu \mathrm{L}$ sterile $0.9 \%$ saline solution. Then both the challenged and control prawns were returned to the PVC tanks of aerated seawater and fed at $25^{\circ} \mathrm{C}$ as described above. The seawater waste was dealt with safely to make it harmless for the environment. Hemocytes and hepatopancreas of six prawns from each treatment (the challenged group and the control group) were randomly sampled at $0,3,6,12,24,48$, and $72 \mathrm{~h}$ post-injection respectively, then the samples were snap-frozen in liquid nitrogen. There were three replicates for each time point. Total RNA was extracted and the first strand cDNA was synthesized as described above.

\section{Expression of EcFAMeT after $V$. anguillarum and WSSV challenge}

Real time quantitative RT-PCR was performed on an ABI PRISM 7500 Sequence Detection System (Applied Biosystems, USA) to investigate the expression of EcFAMeT. For the mRNA expression of EcFAMeT, the pair of specific primers F2 and R2 (Table 1) were used to amplify a PCR product of $593 \mathrm{bp}$. Two primers 18S-HF and 18S-HR (Table 1) were used to amplify an 18S rRNA gene of $147 \mathrm{bp}$ as an internal control to verify the successful reverse transcription and to calibrate the cDNA template. The RT-PCR was carried out in a total volume of $20 \mu \mathrm{L}$, containing $10 \mu \mathrm{L}$ SYBR $^{\circledR}$ Premix Ex Taq ${ }^{\text {TM }}$ II $(2 \times)($ TaKaRa), $2 \mu \mathrm{L}$ of the $1: 5$ diluted cDNA, $0.8 \mu \mathrm{L}$ each of F2 and R2 primer (or 18S-HF and $18 \mathrm{~S}-\mathrm{HR}$ to amplify the $18 \mathrm{~S}$ rRNA), $0.4 \mu \mathrm{L}$ ROX Reference Dye II $(50 \times) * 3$, and $6 \mu \mathrm{L}$ DEPC-treated water. The PCR program was $95{ }^{\circ} \mathrm{C}$ for $30 \mathrm{~s}$, then 40 cycles of $95{ }^{\circ} \mathrm{C}$ for $5 \mathrm{~s}$ and $60{ }^{\circ} \mathrm{C}$ for $34 \mathrm{~s}$, followed by 1 cycle of $95{ }^{\circ} \mathrm{C}$ for $15 \mathrm{~s}, 60^{\circ} \mathrm{C}$ for $1 \mathrm{~min}$ and $95{ }^{\circ} \mathrm{C}$ for $15 \mathrm{~s}$. DEPCtreated water for the replacement of template was used as negative control.

RT-PCR data from three replicate samples were analyzed with the ABI 7300 system SDS Software (Applied Biosystems, USA), for estimating transcript copy numbers for each sample. The comparative $C_{\mathrm{T}}$ method was to analyze the relative expression levels of EcFAMeT. The $C_{\mathrm{T}}$ for the target amplified products of EcFAMeT and internal control 18S rRNA was determined for each sample. The difference in 
the $C_{\mathrm{T}}$ between the target and the internal control, called ${ }^{\Delta} C_{\mathrm{T}}$, was calculated to normalize the differences in the amount of template and the efficiency of the RT-PCR. In the same challenge time, the ${ }^{\Delta} C_{\mathrm{T}}$ of the control group was used as the calibrator, and the difference between the ${ }^{\Delta} C_{\mathrm{T}}$ of the challenged group and the control group was called $\Delta \Delta C_{\mathrm{T}}$. The expression level of EcFAMeT was calculated by the $2^{-\triangle \Delta C T}$ comparative $C_{T}$ method (Livak and Schmittgen 2001). Statistical analysis was performed using SPSS software (Ver 11.0). Statistical significance was determined using one-way ANOVA (González-Rodríguez et al. 2012) and post hoc Duncan multiple range tests. Significance was set at $P<0.05$.

\section{Results}

Analysis of the EcFAMeT sequence and the predicted protein

A full-length 1,620-bp cDNA of FAMeT (designated EcFAMeT) was obtained from the hemocytes of $E$. carinicauda by RACE method, and the nucleotide and deduced amino acid sequences were shown in Fig. 1. It contained an open reading frame (ORF) of $831 \mathrm{bp}, 75 \mathrm{bp}$ of 5 '-untranslated region (UTR), 714 bp of 3'-UTR including a stop codon (TAA) polyadenylation signal (AATAAA) and a poly A tail. The EcFAMeT cDNA sequence has been submitted to the GenBank (GenBank accession number: KF534709).

The ORF of EcFAMeT encoded 276 amino acids without signal peptide analyzed by SignalP software, indicating that EcFAMeT did not belong to the family of secretory proteins. The calculated molecular mass was $31.57 \mathrm{kDa}$, and the estimated isoelectric point was 4.67. InterProScan analysis showed that the amino acid sequences ${ }^{38} \mathrm{H}-\mathrm{W}^{137}$ and ${ }^{174} \mathrm{H}-$ $\mathrm{W}^{272}$ were the putative conserved domains of MethyltransfFA superfamily.

Multiple sequences and phylogenetic analysis

Sequence analysis with the BLASTP program revealed that the deduced amino acid sequence of EcFAMeT exhibited a high identification with FAMeT of Macrobrachium nipponense (90\%), Pandalopsis japonica (83\%), Marsupenaeus japonicus (81\%), Fenneropenaeus chinensis (81\%), Penaeus monodon (81\%), L. vannamei (79\%), M. ensis (79\%), Homarus americanus (79\%), Portunus trituberculatus (78 \%), Cancer pagurus (78\%), Scylla paramamosain (76\%), and Eriocheir sinensis (75\%) counterparts, respectively. Multiple sequence alignment of EcFAMeT with other animal FAMeTs revealed that they were highly conserved, especially in the regions of FAMeT family signatures. However, some shrimp FAMeTs consist of three or five additional amino acids in the amino acid sequences (Fig. 2).

Based on the amino acid sequences of FAMeTs, a NeighborJoining phylogenetic tree was constructed using MEGA 4.0 for phylogenetic analysis (Fig. 3). Shrimp and crab FAMeTs were separated and formed two distinct branches in the tree. In the branch of shrimp, all the shrimps FAMeTs were clustered together and formed three branches. All the FAMeTs of penaeid shrimp were clustered together and formed a subgroup, and FAMeTs of H. americanus, Procambarus clarkia, and Cherax quadricarinatus were classified into another subgroup. EcFAMeT of E. carinicauda showed closer evolutionary

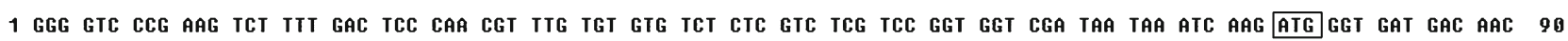

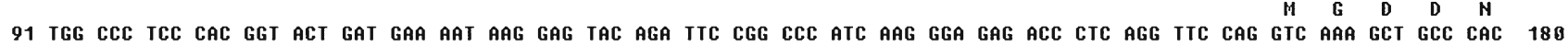

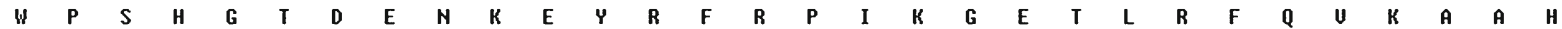

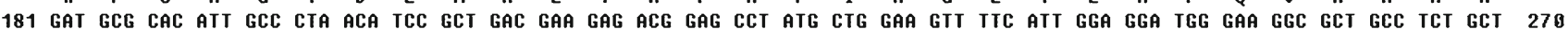
D $A \begin{aligned} & H \\ & A\end{aligned}$

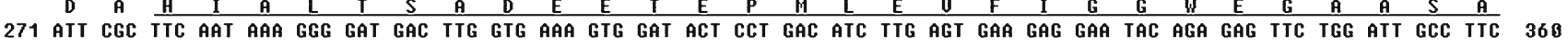

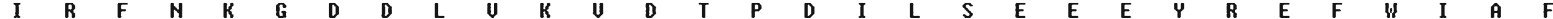
361 GAT CAC GGT GAA ATC AGA GTC GGG AAA GGT GGA GAG TGG GAG CCA CTC ATG ATG TGC CCT ATI CCA GAA CCT ITT GGA ATC ACC CAC TIT 450

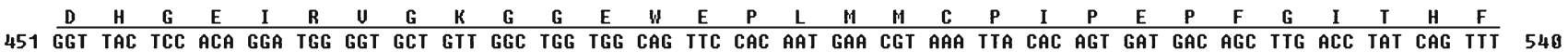

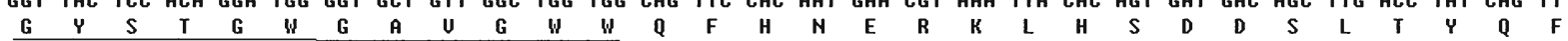

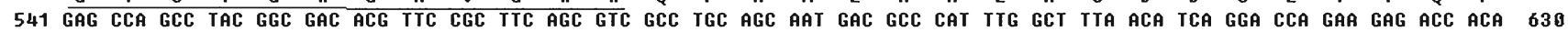

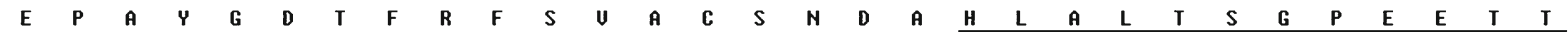

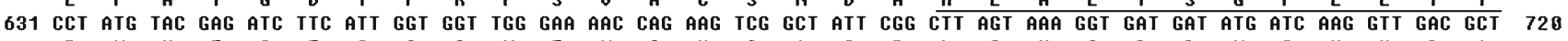

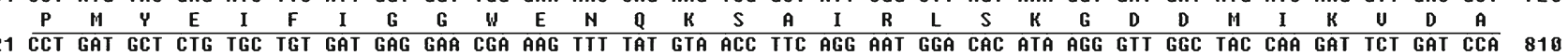

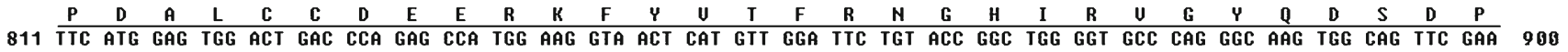

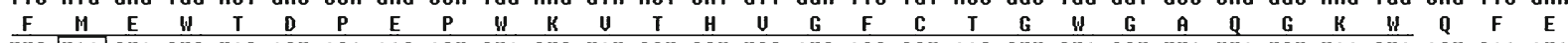

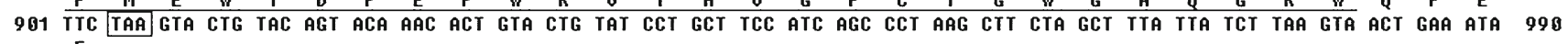
$F \stackrel{2}{*}$

991 IIT ITA TAC GCC IGT TGA TCA TAG CAT AAG AAA CAC CGG TAG ITA AAT ITT TAG ITG AGC ITG GTG ACT GCT ITG AGG AAT GGT ITC ICT 1089 1981 CAA ATT CIT GAA TAT AGA ATT AAG AAA CIT ATG TCA TIT AGT GTC ITC ATT CCT CCT ITG TGG TIT TCA ITT AGG TCC ATG TCT GTA AGC 117G 1171 CAT TGC CTC AAT TTG TCA AAT TTA ATG TCT GCA CTG ACC AGT AGT GCC GTA AAT CCT TTG TTG GCT GGT GTC CGG AGG TTT CTC CTT TAT 126B 1261 GAA ATT ITG CAA ACC ACC TAT ITG ITT AAG GCT TIC TAT TGT AAA GAA AAA ATA AGC ITA GCT TIT IGG TGT AAT TTA GAG ATC TGT TAG 135B 1351 CCA TCT CCA GTT ITG TGA AAT TIG AAA ATT GGA TAT GTA CTG TAA AGC ATA CAT TCA GGT TCA TTA TCT TTG GIT CTA TAT TGC AAT TTA 1440 1441 TCA ACT TAA ATT TTG ACT GTT TTG ATT TTG AAT ATT TTA ATA AAT GTT TGA AGA GAT CAA AGA AAG TAC TTT AAT GCA TTA ITA GAA AGT 153G

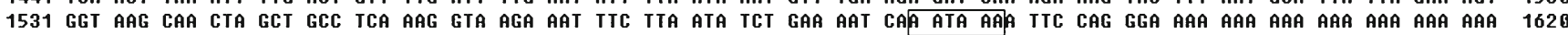

Fig. 1 Nucleotide and deduced amino acid sequences of EcFAMeT cDNA of E. carinicauda. The letters in the box indicated the start codon (ATG), the polyadenylation signal sequence (AATAAA), and the stop codon (TAA). The two FAMeT superfamily signature motifs were underlined 
E. carinicauda FAMeT

M. nipponense F AMeT

$F$. chinensis FaMeT

L. vannamei FAMET-S

L. vanname i FAMET-L

P. monodon F AMeT-S

P. monodone FAMeT-L

$\mathrm{F}$. merguiensis FAMeT-S

F, merguiensis FHMeT-L

s. paramamosain FAMeT-S

5. paramamosain FAMET-I

. paramamosain FAMeT-L

serrata F AMET-S

s. serrata FAMeT-I

s. serrata FAMeT-L

P. pelagicus FaMeT-S

P. pelagicus FAMeT-I

$P$. pelagicus FAMeT-L

E. carinicauda FAMeT

M. nipponense FaMeT

F. chinensis FAMeT

L. vannamei FAMeT-S

L. vannamei FAMeT-L

P. monodon FÀMeT-S

P. monodone FAMeT-L

F. merguiensis F.MeT-S

F. merguiensis FAMeT-L

S. paramamosain FAMET-S

s. paramamosain FAMeT-I

paramamosain $\mathrm{F}$ AMe $\mathrm{T}-\mathrm{L}$

- serrata FAMeT-S

- serrata FAMeT-I

. serrata FaMeT-L

P. pelagicus FAMeT-S

P. pelagicus FAMeT-I

P. pelagicus FAMeT-L

E. carinicauda FAMET

M. nipponense FAMET

$F$. chinensis FAMeT

L. vannamei FoMeT-S

L. vannamei FAMeT-L

P. monodon FAMET-S

P. monodone F.MeT-L

F. merguiensis FAMeT-S

F. merguiensis FAMET-L

S. paramamosain FAMeT-S

. paramamosain FoMeT-I

s. paramamosain FAMET-L

s. serrata FAMeT-s

S. serrata $F$ HMeT-I

s. serrata FaMeT-L

P. pelagicus FaMeT-S

P. pelagicus FAlleT-I

P. pelagicus FAMeT-L

Fig. 2 Multiple alignment of EcFAMeT with other known FAMeTs: $M$. nipponense FAMeT (AFA26604); F. chinensis FAMeT (AFA28125); L. vannamei: FAMeT-S (AAZ22180), FAMeT-L (AAZ22181); P. monodon FAMeT-S (ABA86955), FAMeT-L (ABA86956); F. merguiensis FAMeT-S (ABA86957), FAMeT-L (ABA86958); C. quadricarinatus FAMeT-S (ABA86959), FAMeT-L (ABA86960); S. paramamosain

relationships with FAMeT of $M$. nipponense. The relationships displayed in the phylogenetic tree were in good agreement with the concept of traditional taxonomy.

Tissue expression of EcFAMeT gene

To investigate the expression profiles of EcFAMeT, quantitative real-time RT-PCR was employed to analyze their relative expression levels in different tissues, including hemocytes, gill, hepatopancreas, muscle, ovary, intestine, stomach, and eyestalk.
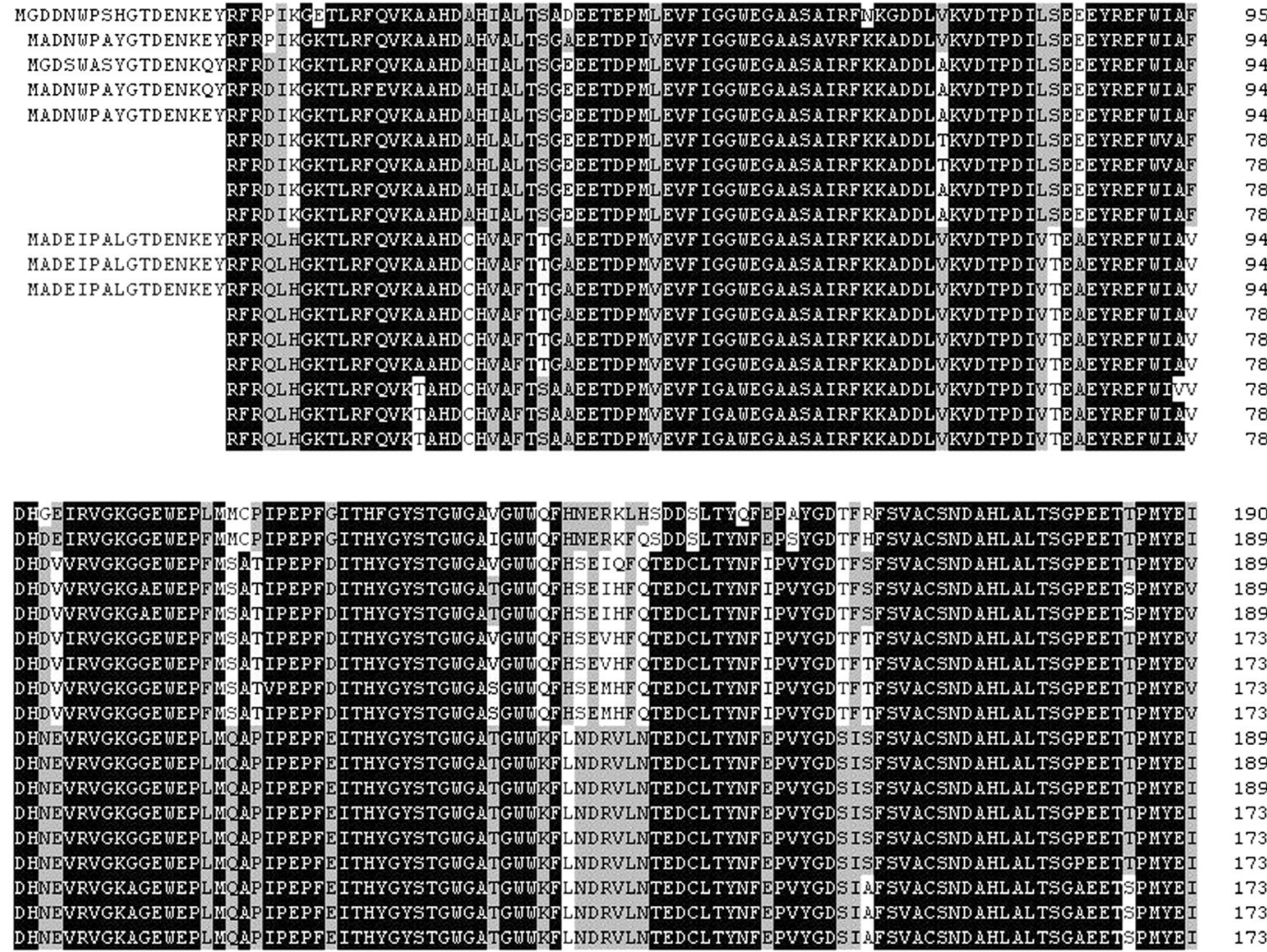

173

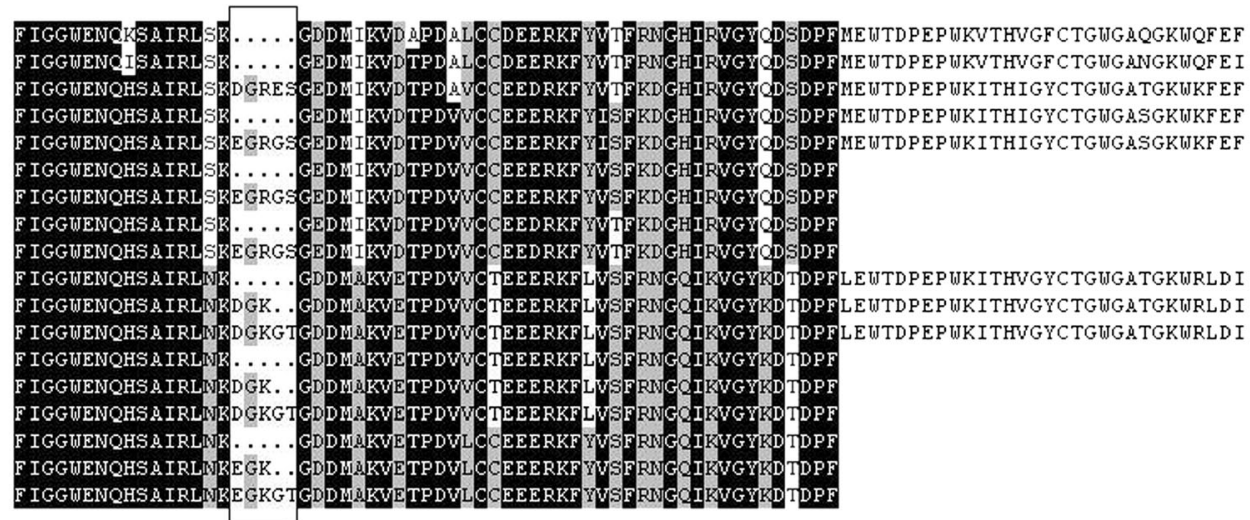

276

FAMeT-S (AEE26197), FAMeT-I (ADK32330), FAMeT-L (AEE26196); Scylla serrata: FAMeT-S (ABA86954), FAMeT-I (ABA86953), FAMeT-L (ABA86952); P. pelagicus FAMeT-S (AAZ40196), FAMeT-I (AAZ40197), FAMeT-L (ABB22042). The inserted amino acids in various FAMeT informs were marked by frame

The mRNA transcripts of EcFAMeT could be detected in all the tested tissues, but showed different expression levels. EcFAMeT was highly expressed in hepatopancreas, followed by ovary, and the lowest expression was in intestine (Fig. 4).

Expression profiles of EcFAMeT in hemocytes and hepatopancreas after $V$. anguillarum challenge

Expression profiles of EcFAMeT mRNA in hemocytes and hepatopancreas of E. carinicauda after $V$. anguillarum 
Fig. 3 Phylogenetic tree of different species FAMeTs on the basis of the amino acid sequence using neighbor-joining distance analysis. The protein sequences used for phylogenetic analysis were as follows: FAMeT: $M$. nipponense (AFA26604), $F$. chinensis (AFA28125), $P$. japonica (ADR64207), M. japonicas (BAE78496), $H$. americanus (AAA67080), $M$. ensis (AAK28535), C. pagurus (AAR00732), P. trituberculatus (AGH25488), E. sinensis (ACX30003), P. clarki (AEB54633); L. vannamei FAMeT-S (AAZ22180), FAMeTL (AAZ22181); P. monodon FAMeT-S (ABA86955), FAMeTL (ABA86956); F. merguiensis: FAMeT-S (ABA86957), FAMeT-L (ABA86958); $C$. quadricarinatus FAMeT-S (ABA86959), FAMeT-L (ABA86960); S. paramamosain FAMeT-S (AEE26197), FAMeT-I (ADK32330), FAMeT-L (AEE26196); S. serrata FAMeTS (ABA86954), FAMeT-I (ABA86953), FAMeT-L (ABA86952); P. pelagicus FAMeT-S (AAZ40196), FAMeTI (AAZ40197), FAMeT-L (ABB22042). The numbers at the forks indicated the bootstrap

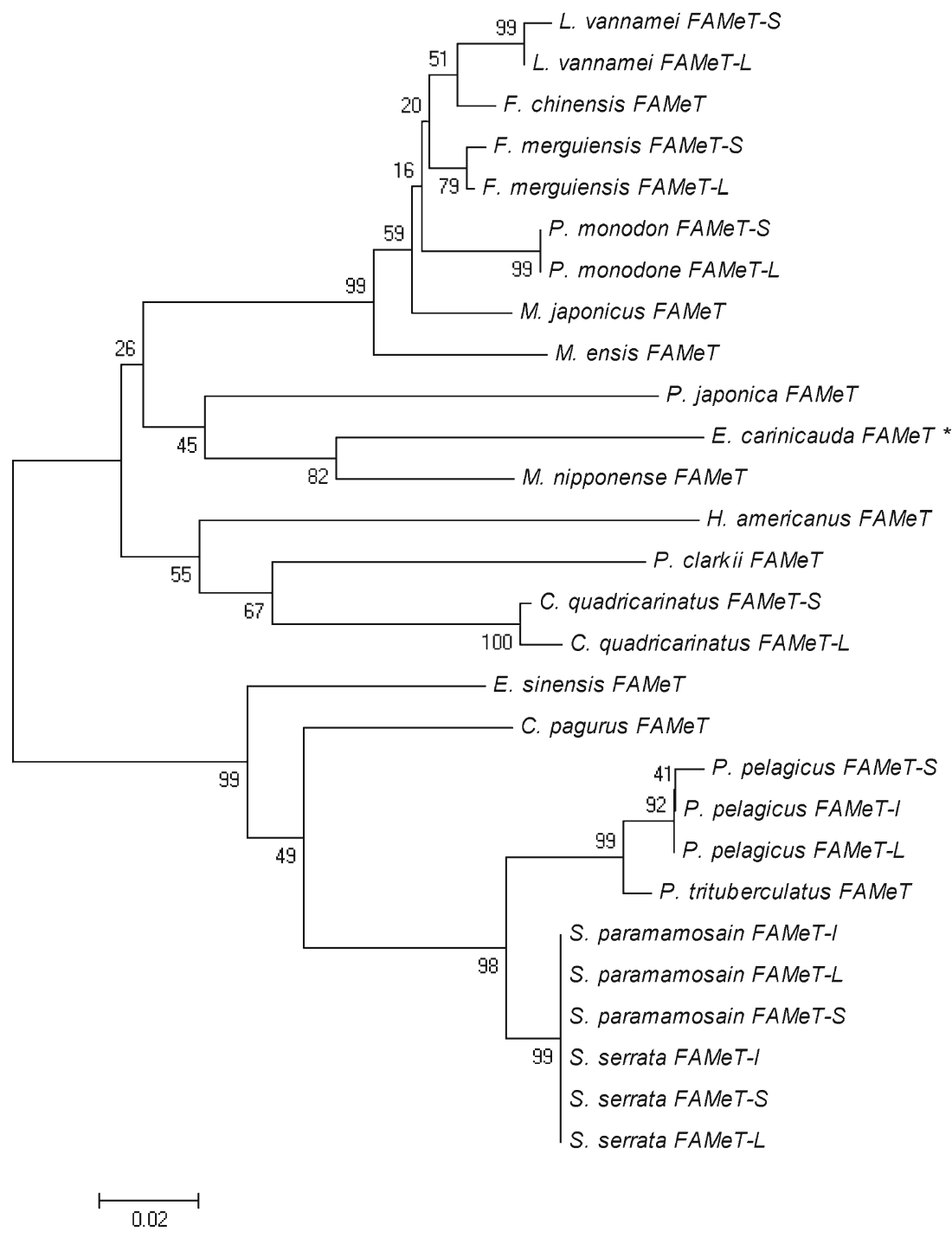

injection were shown in Fig. 5. Compared to the control, the expression of EcFAMeT in hemocytes increased significantly and reached a peak value at $6 \mathrm{~h}$ (3.87-fold of the control group, $P<0.05)$, then it decreased gradually and dropped to the lowest level at $48 \mathrm{~h}(0.13$-fold of the control group, $P<0.05)$, then it upregulated to the control group level at $72 \mathrm{~h}$ (Fig. 5a). EcFAMeT transcripts in hepatopancreas obviously increased to the first peak level at $3 \mathrm{~h}$ (2.94-fold of the control group, $P<0.05)$, after decreasing significantly from $3 \mathrm{~h}$ to $6 \mathrm{~h}$ (0.32-fold of the control group, $P<0.05)$, it up-regulated again to the second peak level at $12 \mathrm{~h}$ (4.64-fold of the control group, $P<0.05)$, then it decreased gradually and recovered to the control level at $72 \mathrm{~h}$ (Fig. 5b).

Expression profiles of EcFAMeT in hemocytes and hepatopancreas after WSSV challenge

The temporal expression of EcFAMeT mRNA in hemocytes and hepatopancreas of $E$. carinicauda after WSSV challenge was shown in Fig. 6. Compared with the control group, EcFAMeT transcripts in hemocytes were significantly induced by WSSV challenge, and reached to the peak level at $6 \mathrm{~h}$ (3.32-fold of the control group, $P<0.05$ ), then dropped to the lowest level at $12 \mathrm{~h}$ (0.27-fold of the control group, $P<0.05)$. Afterwards, the transcript level of EcFAMeT increased gradually and reached the control level at $72 \mathrm{~h}$ (Fig. 6a). In hepatopancreas, the EcFAMeT mRNA expression level increased significantly and reached to the peak level at $3 \mathrm{~h}(2.89$-fold of the control group, $P<0.05)$, after a significant decrease at $6 \mathrm{~h}$ (0.14-fold of the control group, $P<0.05)$, it increased again to the second peak level at $12 \mathrm{~h}$ postchallenge (1.80-fold of the control group, $P<0.05)$. Afterwards, the EcFAMeT mRNA expression level decreased significantly at $24 \mathrm{~h}$, and increased gradually to the control level at $72 \mathrm{~h}$ (Fig. 6b).

\section{Discussion}

Invertebrates lack an adaptive immune system and their defense mechanisms mainly rely on innate immune responses 
Fig. 4 Tissue specific expression of EcFAMeT mRNA related to hepatopancreas expression by the real-time PCR. The reference gene is $18 \mathrm{~S}$ rRNA. Vertical bars represent the mean $\pm \mathrm{SD}(N=3)$. Significant differences $(P<0.05)$ in EcFAMeT expression between different tissues were indicated with different letters

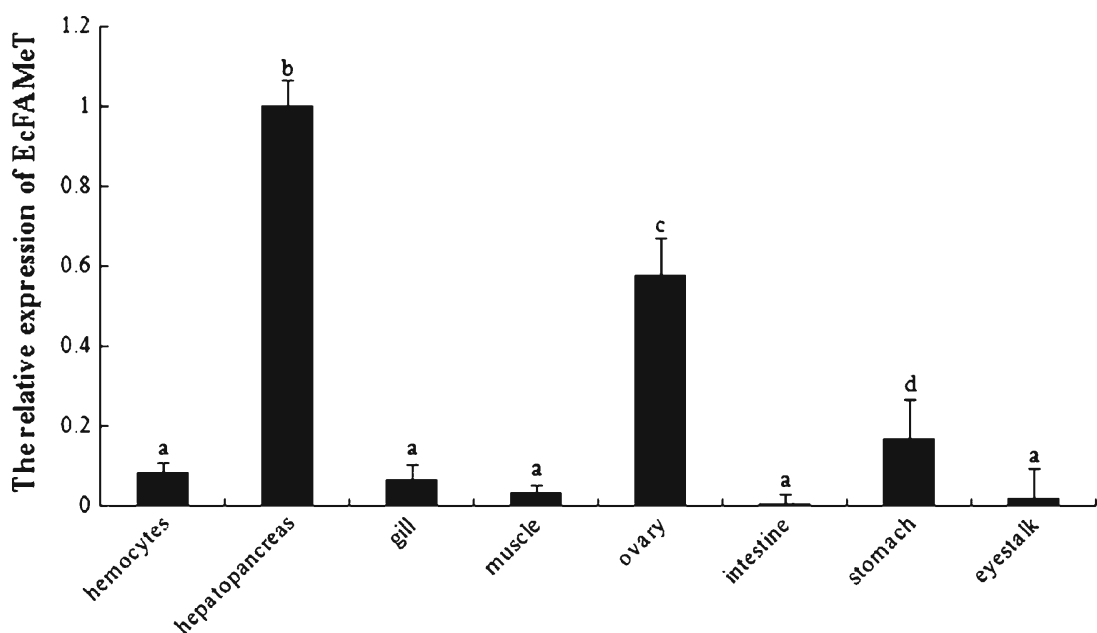

The analyzed tissues for protecting them against invaders (Zhang et al. 2008; Iwanaga and Lee 2005). The identification and characterization of genes involved in immune responses are essential for the elucidation of immune defense mechanisms and for disease control (Zhao et al. 2007). In the present study, a new full-length 1,620-bp of FAMeT cDNA (EcFAMeT) was isolated and sequenced from E. carinicauda. The EcFAMeT sequence showed a high identification (75-90 \%) with FAMeTs of other crustaceans. Multiple sequence alignment analysis revealed that the FAMeT sequences were highly conserved. Phylogenetic analysis revealed that EcFAMeT of E. carinicauda was clustered with FAMeT of other crustaceans. The sequence alignment and phylogenetic analysis further suggested that EcFAMeT was a member of the FAMeT family and should have the same function as FAMeT from other species due to the conserved sequences and motifs.

In penaeid shrimp L. vannamei, P. monodon, and Fenneropenaeus merguiensis, two different isoforms of FAMeT (long and short isoform) were found which were named FAMeT-L and FAMeT-S respectively, and they were almost identical except for the additional five amino acids in the FAMeT-L isoform (Fig. 2). In the crabs $S$. serrata, $S$. paramamosain, and Portunus pelagicus, three different isoforms (long, intermediate and short isoform) were found in the amino acid sequence of FAMeT, and there were three or five different amino acids between the various types of isoform. EcFAMeT lacked the five inserted amino acids, indicating that EcFAMeT was more closely related to the FAMeT short isoform (FAMeT-S) of other crustaceans (Fig. 2). But in our study, as there were no other isoforms of FAMeT found in E. carinicauda, it might be related to the total RNA extracted from different tissues in the process of gene cloning. We will extract total RNA from different tissues in the future, to prove whether there are other isoforms of FAMeT in E. carinicauda.
FAMeT is one of the most abundant cellular proteins and multiple tissues of crustaceans contain RNA transcripts (Ruddell et al. 2003; Silva Gunawardene et al. 2001, 2002). In our study, tissue-specific differences in levels of constitutive FAMeT were observed. The EcFAMeT mRNA level was high in hepatopancreas and ovary as compared with other six tested tissues (hemocytes, gill, muscle, stomach, intestine, and eyestalk), which implied that EcFAMeT might have a certain relationship with metabolism, genital organ development, and maturation. Previous studies have demonstrated that FAMeT might be involved in many important biological functions in crustaceans, including metamorphosis, reproduction, and molting (Holford et al. 2004; Silva Gunawardene et al. 2001; Averbeck et al. 2000). In the insects Manduca sexta, Helicoverpa zea, and Vanessa cardui, only the reproductive tract of males contained the FAMeT, but the female had none, indicating that FAMeT was a sex-specific expression gene (Bhaskaran et al. 1988). In the eyestalk of $M$. ensis, FAMeT was expressed in the adult shrimp during all stages of reproductive development; whereas in the juveniles, it was only expressed in male shrimp and not in the females, which showed that FAMeT might be important for the regulation of eyestalk neuropeptides and involved in the regulation of the reproduction and molting processes in crustaceans through the synthesis of MF (Silva Gunawardene et al. 2001). After double stranded RNA (dsRNA) injection, the expression of FAMeT in the eyestalk of L. vannamei was knocked down and the shrimp did not advance to the final stage of the molt cycle. Moreover, the molt-related genes (cathepsin-L and hemocyanin) were disturbed and a $100 \%$ mortality of the shrimp was observed, all of which revealed that FAMeT might be involved in the control of molting in shrimp (Hui et al. 2008). A similar conclusion was also reached in $S$. paramamosain (Yang et al. 2012). Many studies focus principally on the function of FAMeT in the reproduction and 
Fig. 5 The mRNA expression levels of EcFAMeT in hemocytes (a) and hepatopancreas (b) of E. carinicauda at different time intervals after $V$. anguillarum challenge treatment. The reference gene is $18 \mathrm{~S}$ rRNA. Vertical bars represented the mean $\pm \mathrm{SD}(N=3)$. Significant differences $(P<0.05)$ in

EcFAMeT expression between the challenged and the control group were indicated with asterisks
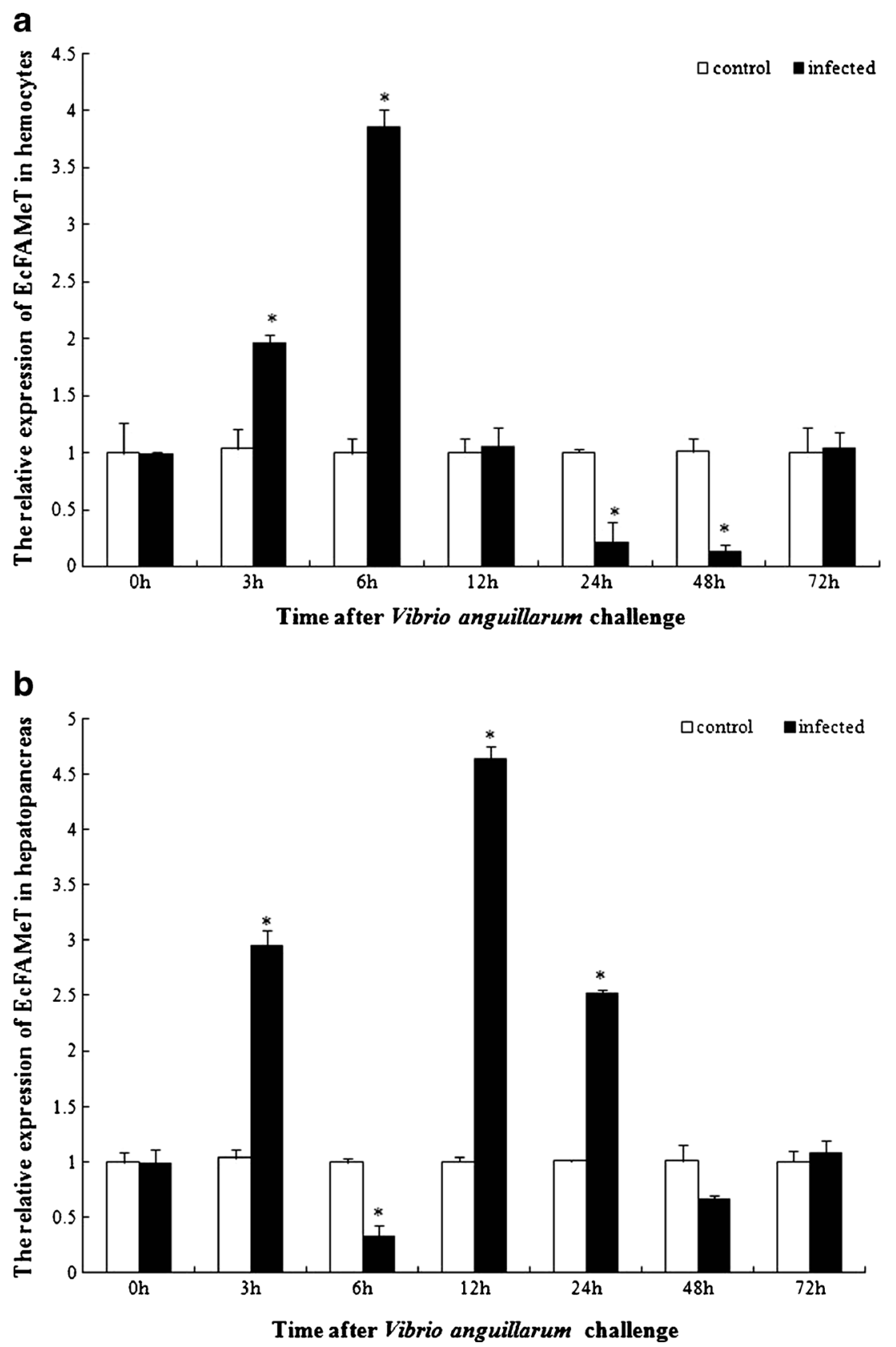

molting processes, but whether FAMeT is related to the immune response of shrimp has not yet been studied.

Virulent pathogens have a major impact on aquaculture. $V$. anguillarum and WSSV are both extremely virulent pathogens that seriously affect shrimp aquaculture (Toranzo et al. 2005; Lightner 2011; Spann and Lester 1997). When pathogens enter the body of the shrimp, they will encounter the innate immune system (Soonthornchai et al. 2010). Previous studies have demonstrated that hemocytes and hepatopancreas are the main cells and tissue involved in the immune response, and the major site for the synthesis of immune defense molecules involved in eliminating pathogens or other particulate matter in crustaceans. Information on the expression profile of the EcFAMeT gene in these tissues after $V$. anguillarum and WSSV challenge would be helpful to better understand its immunological function.

$V$. anguillarum is a major virulent pathogen for cultured shrimp worldwide (Toranzo et al. 2005). As far as we know, the effect of $V$. anguillarum on the expression of FAMeT has not been evaluated in crustaceans. In the present study, live $V$. anguillarum were chosen to challenge the shrimp, so that the shrimps' health would be affected severely by the production 
Fig. 6 The mRNA expression levels of EcFAMeT in hemocytes (a) and hepatopancreas (b) of E. carinicauda at different time intervals after WSSV challenge treatment. The reference gene is 18S rRNA. Vertical bars represented the mean $\pm \mathrm{SD}(N=3)$. Significant differences $(P<0.05)$ in EcFAMeT expression between the challenged and the control group were indicated with asterisks
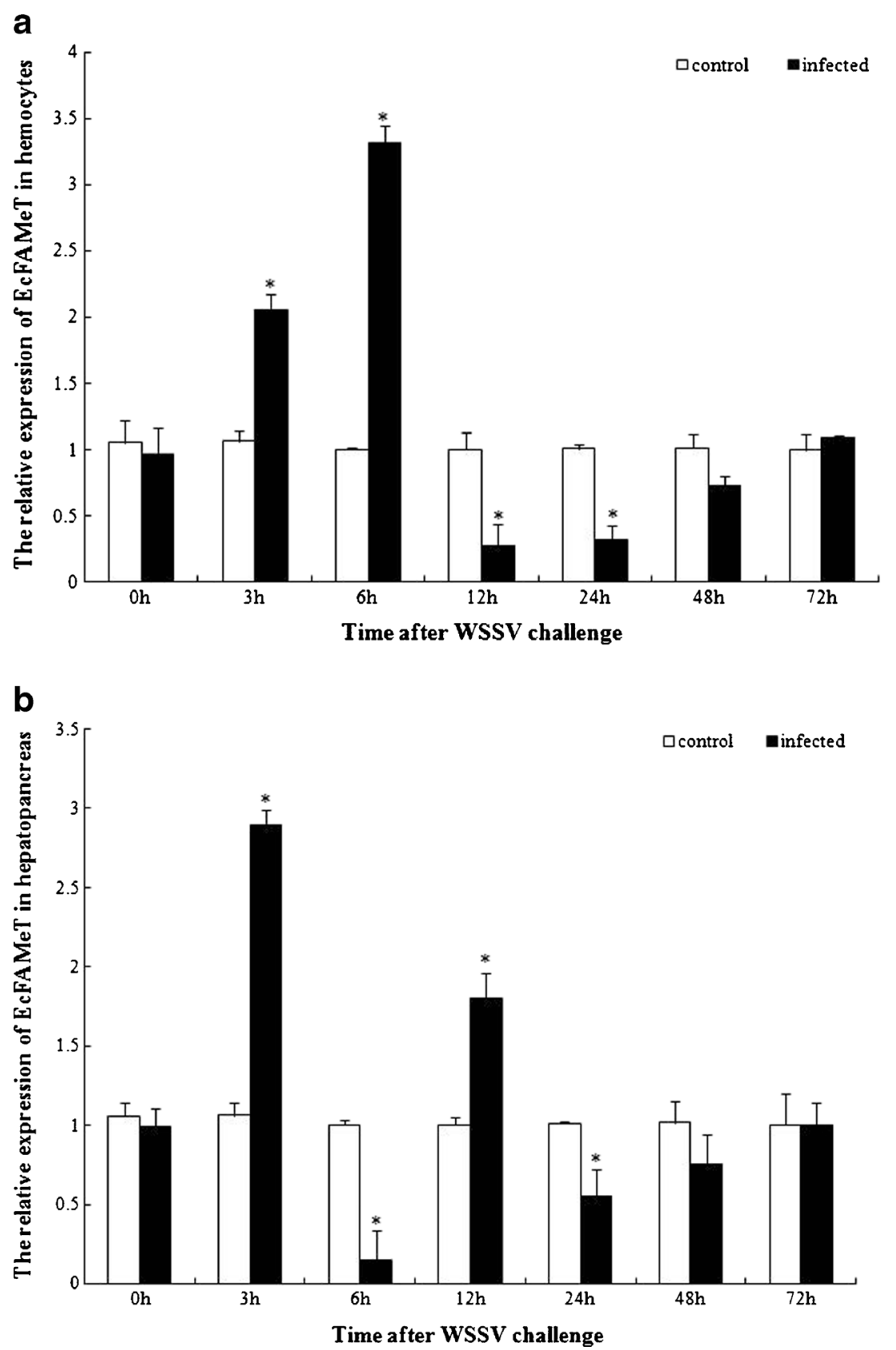

of $V$. anguillarum. When injected with mixtures of $V$. anguillarum and Staphylococcus aureus, the expression level of OMT was up-regulated in the hepatopancreas and hemocytes of $F$. chinensis (Li et al. 2006). In our study, the expression of EcFAMeT increased at $3 \mathrm{~h}$ significantly in hemocytes and hepatopancreas respectively, which indicated that EcFAMeT could be induced by $V$. anguillarum. As time progressed, the expression of EcFAMeT dropped to a low level at $48 \mathrm{~h}$ in both hemocytes and hepatopancreas, perhaps because the infection progress brought more bacteria, and destroyed the normal function of the shrimps' cells and finally caused the expression of EcFAMeT in the challenged group to decrease gradually (Chen et al. 2011). The results showed that EcFAMeT might be involved in an immune response to $V$. anguillarum stimulation. The expression profiles of the EcFAMeT gene with $V$. anguillarum challenge indicated that it was inducible and might be involved in the immune response against bacterial infection in E. carinicauda.

White spot syndrome (WSS) is a lethal disease that affects cultured shrimp species (Lightner 2011). WSSV is a large DNA virus with a broad host range among shrimps and the causative agent of White Spot disease of shrimp, causing mass mortalities and economic losses in shrimp aquaculture (Lightner 2011; Escobedo-Bonilla et al. 2008; Leu et al. 2009). 
However, there are still no effective preventive and therapeutic measures against WSSV infection. In our study, the transcriptional profile of EcFAMeT was analyzed in hemocytes and hepatopancreas of E. carinicauda when shrimps were injected with WSSV. The transcript levels of EcFAMeT following the WSSV injection ascended at $6 \mathrm{~h}$ in hemocytes and at $3 \mathrm{~h}$ in hepatopancreas, respectively, demonstrating that it might play important roles in the immune response against WSSV infection in shrimp. The data indicated that the expression profiles of EcFAMeT were very similar when shrimp were challenged by $V$. anguillarum and WSSV. But whether in the $V$. anguillarum challenged group or in the WSSV challenged group, the expression profiles of EcFAMeT in hemocytes were different with hepatopancreas, which might be caused by the different function of hemocytes and hepatopancreas in the immune defense system. It has been proved that hemocytes are key cells for invertebrates' innate defense reactions (Vazquez et al. 2009; Iwanaga and Lee 2005) and play an important role in the host immune functions when the organism is attacked by bacterias or viruses (Lee and Söderhäll 2002; Lavine and Strand 2002). In the presence of stressors such as elevated temperature, anoxia and diluted seawater, the hemolymph titer of MF in the crab increased (Lovett et al. 2001; Borst et al. 2001).

Acknowledgments The authors are grateful to all the laboratory members for experimental material preparation and technical assistance. This study was supported by the earmarked fund for National "863" Project of China (No. 2012AA10A409), Modern Agro-industry Technology Research System (No. CARS-47), Special Fund for Agro-scientific Research in the Public Interest (No. 201103034), and in part by the Special Scientific Research Funds for Central Non-profit Institutes, Chinese Academy of Fishery Sciences (2013A0701).

Open Access This article is distributed under the terms of the Creative Commons Attribution License which permits any use, distribution, and reproduction in any medium, provided the original author(s) and the source are credited.

\section{References}

Averbeck NB, Jensen ON, Mann M, Schagger H, Osiewacz HD (2000) Identification and characterization of PaMTH1, a putative Omethyltransferase accumulating during senescence of Podospora anserina cultures. Curr Genet 37:200-208

Bhaskaran G, Sparagana SP, Dahm KH, Barrera SP, Peck K (1988) Sexual dimorphism injuvenile hormone synthesis by Corpora allata and in juvenile hormone acid methyltransferrase activity in Corpora allata and accessory sex glands of some Lepidoptera. Int J Invertebr Reprod Dev 13:87-100

Borst DW, Ogan JT, Tsukimura B, Claerhout T, Holford KC (2001) Regulation of the crustacean mandibular organ. Am Zool 41:430 441

Burtenshaw SM, Su PP, Zhang JR, Tobe SS, Dayton L, Bendena WG (2008) A putative farnesoic acid O-methyltransferase (FAMeT) orthologue in Drosophila melanogaster (CG10527): Relationship to juvenile hormone biosynthesis? Peptides 29:242-251
Chen P, Li JT, Gao BQ, Liu P, Wang QY, Li J (2011) cDNA cloning and characterization of peroxiredoxin gene from the swimming crab Portunus trituberculatus. Aquaculture 322-323:10-15

Chiron H, Drouet A, Claudot AC, Eckerskrn C, Trost M, Heller W, Ernst D, Sandermann HJ (2000) Molecular cloning and functional expression of a stress-induced multifunctional O-methyltransferase with pinosylvin methyltransferase activity from Scots pine (Pinus sylvestris L.). Plant Mol Biol 44:733-745

Duan YF, Liu P, Li JT, Li J, Chen P (2013a) Expression profiles of selenium dependent glutathione peroxidase and glutathione S-transferase from Exopalaemon carinicauda in response to Vibrio anguillarum and WSSV challenge. Fish Shellfish Immunol 35:661-670

Duan YF, Liu P, Li JT, Li J, Chen P (2013b) Immune gene discovery by expressed sequence tag (EST) analysis of hemocytes in the ridgetail white prawn Exopalaemon carinicauda. Fish Shellfish Immunol 34: 173-182

Escobedo-Bonilla CM, Alday-Sanz V, Wille M, Sorgeloos P, Pensaert MB, Nauwynck HJ (2008) A review on the morphology, molecular characterization, morphogenesis and pathogenesis of white spot syndrome virus. J Fish Dis 31:1-18

Fan JB, Zhang CS, Gu NF, Li XW, Sun WW, Wang HY, Feng GY, Clair DS, He L (2005) Catechol-O-methyltransferase gene Val/Met functional polymorphism and risk of schizophrenia: a large-scale association study plus meta-analysis. Biol Psychiatry 57:139-144

González-Rodríguez G, Colubi A, Gil MÁ (2012) Fuzzy data treated as functional data: A one-way ANOVA test approach. Comput Stat Data Anal 56:943-955

Holford KC, Edwards KA, Bendena WG, Tobe SS, Wang ZW, Borst DW (2004) Purification and characterization of a mandibular organ protein from the American lobster, Homarus americanus: a putative farnesoic acid O-methyltransferase. Insect Biochem Mol Biol 34: $785-798$

Hui JHL, Tobe SS, Chan SM (2008) Characterization of the putative farnesoic acid O-methyltransferase (LvFAMeT) cDNA from white shrimp, Litopenaeus vannamei: Evidence for its role in molting. Peptides 29:252-260

Iwanaga S, Lee BL (2005) Recent advances in the innate immunity of invertebrate animals. J Biochem Mol Biol 38:128-150

Kuballa AV, Guyatt K, Dixon B, Thaggard H, Ashton AR, Paterson B, Merritt D, Elizur A (2007) Isolation and expression analysis of multiple isoforms of putative farnesoic acid O-methyltransferase in several crustacean species. Gen Comp Endocrinol 150(1):48-58

Lavine MD, Strand MR (2002) Insect hemocytes and their role in immunity. Insect Biochem Mol Biol 32:1295-1309

Lee S, Söderhäll K (2002) Early events in crustacean innate immunity. Fish Shellfish Immunol 12:421-437

Leu JH, Yang F, Zhang X, Xu X, Kou GH, Lo CF (2009) Whispovirus. Curr Top Microbiol Immunol 328:197-227

Li DX, Du XJ, Zhao XF, Wang JX (2006) Cloning and expression analysis of an O-methyltransferase (OMT) gene from Chinese shrimp, Fenneropenaeus chinensis. Fish Shellfish Immunol 21: 284-292

Li JT, Han JY, Chen P, Chang ZQ, He YY, Liu P, Wang QY, Li J (2012) Cloning of a heat shock protein 90 (HSP90) gene and expression analysis in the ridgetail white prawn Exopalaemon carinicauda. Fish Shellfish Immunol 32:1191-1197

Lightner DV (2011) Virus diseases of farmed shrimp in the Western Hemisphere (the Americas): A review. J Invertebr Pathol 106:110 130

Livak KJ, Schmittgen TD (2001) Analysis of relative gene expression data using real-time quantitative PCR and the 2 (delta delta $\mathrm{C}(\mathrm{T})$ ) method. Methods 25:402-408

Lovett DL, Verzi MP, Clifford PD, Borst DW (2001) Hemolymph levels of methyl farnesoate increase in response to osmotic stress in the green crab, Carcinus maenas. Comp Biochem Physiol A 128:299-306 
Ruddell CJ, Wainwright G, Geffen A, White MRH, Webster SG, Rees $\mathrm{HH}$ (2003) Cloning, characterization, and developmental expression of a putative farnesoic acid O-methyltransferase in the female edible crab Cancer pagurus. Biol Bull 205(3):308-318

Silva Gunawardene YIN, Chow BKC, He JG, Chan SM (2001) The shrimp FAMeT cDNA is encoded for a putative enzyme involved in the methylfarnesoate (MF) biosynthetic pathway and istemporally expressed in the eyestalk of different sexes. Insect Biochem Mol Biol 31:1115-1124

Silva Gunawardene YIN, Tobe SS, Bendena WG, Chow BKC, Yagi KJ, Chan SM (2002) Function and cellular localization of farnesoic acid O-methyltransferase (FAMeT) in the shrimp, Metapenaeus ensis. Eur J Biochem 269:3587-3595

Silva Gunawardene YIN, Bendena WG, Tobe SS, Chan SM (2003) Comparative immunohistochemistry and cellular distribution of farnesoic acid O-methyltransferase in the shrimp and the crayfish. Peptides 24:1591-1597

Söderhäll K, Smith VJ (1983) Separation of the haemocyte populations of Carcinus maenas and other marine decapods. Dev Comp Immunol 7:229-239

Soonthornchai W, Rungrassamee W, Karoonuthaisiri N, Jarayabhand P, Klinbunga S, Söderhäll K, Jiravanichpaisal P (2010) Expression of immune-related genes in the digestive organ of shrimp, Penaeus monodon, after an oral infection by Vibrio harveyi. Dev Comp Immunol 34:19-28

Spann KM, Lester RJG (1997) Special topic review: viral diseases of penaeid shrimp with particular reference to four viruses recently found in shrimp from Queensland. World J Microbiol Biotechnol 13:419-426

Tamura K, Dudley J, Nei M, Kumar S (2007) MEGA 4: molecular evolutionary genetics analysis (MEGA) software version 4.0. Mol Biol Evol 24:1596-1599

Toranzo AE, Magarinos B, Romalde JL (2005) A review of the main bacterial fish diseases in mariculture systems. Aquaculture 246:37-61
Vazquez L, Alpuche J, Maldonado G, Agundis C, Pereyra-Morales A, Zenteno E (2009) Immunity mechanisms in crustaceans. Innate Immunol 15:179-188

Wang LY, Li FH, Wang B, Xiang JH (2013) A new shrimp peritrophinlike gene from Exopalaemon carinicauda involved in white spot syndrome virus (WSSV) infection. Fish Shellfish Immunol 35:840 846

Williamson M, Lenz C, Winther AM, Nassel DR, Grimmelikhuijzen CJ (2001) Molecular cloning, genomic organization, and expression of a B-type (cricket-type) allatostatin preprohormone from Drosophila melanogaster. Biochem Biophys Res Commun 281: $544-550$

Xie XX, Li HY, Xu LM, Yang F (2005) A simple and efficient method for purification of intact white spot syndrome virus (WSSV) viral particles. Virus Res 108:63-67

$\mathrm{Xu}$ WJ, Xie JJ, Shi H, Li CW (2010) Hematodinium infections in cultured ridgetail white prawns, Exopalaemon carinicauda, in eastern China. Aquaculture 300:25-31

Yang YN, Ye HH, Huang HY, Jin ZX, Li SJ (2012) Cloning, expression and functional analysis of farnesoic acid O-methyltransferase (FAMeT) in the mud crab, Scylla paramamosain. Mar Freshw Behav Physiol 45(3):209-222

Yao ZJ, Zhang ZJ, Zhu RX, Mou XD, Chen JF, Sun J (2003) Association of negative symptoms with catechol-O-methyl transferase functional polymorphism in untreated schizophrenic patients. J Clin Psychol Med 13:321-324 [In Chinese]

Zhang QL, Li FH, Zhang XJ, Dong B, Zhang JQ, Xie YS, Xiang JH (2008) cDNA cloning, characterization and expression analysis of the antioxidant enzyme gene, catalase, of Chinese shrimp Fenneropenaeus chinensis. Fish Shellfish Immunol 24:584-591

Zhao JM, Song LS, Li CH, Ni DJ, Wu LT, Zhu L, Wang H, Xu W (2007) Molecular cloning, expression of a big defensin gene from bayscallop Argopecten irradians and the antimicrobial activity of its recombinant protein. Mol Immunol 44:360-368 